\title{
Nobel Lecture: LIGO and the discovery of gravitational waves I
}

\author{
Rainer Weiss \\ Massachusetts Institute of Technology (MIT), Cambridge, Massachusetts 02139, USA
}

(published 18 December 2018)

DOI: 10.1103/RevModPhys.90.040501

\section{CONTENTS}

References

All three of us-Barry Barish, Kip Thorne and I-want to recognize the critical role played by the scientists, engineers, students, technicians and administrators of the Laser Interferometer Gravitational-wave Observatory (LIGO) laboratory and LIGO Scientific Collaboration who are responsible for opening a new field of scientific research: Gravitational Wave Astronomy and Astrophysics. We are also deeply indebted to the United States National Science Foundation, which was willing to take a risk in supporting a new field that required significant technical development and with an uncertain knowledge of sources but certain that, should it succeed, it would have a profound influence on our understanding of physics and the universe.

The three of us will give talks with the same title, "LIGO and the Discovery of Gravitational Waves," but focus on different aspects. I will discuss some of the early history of gravitational waves and develop the concepts to understand the detectors as well as the challenges faced in measuring strains as small as $10^{-21}$. Barry will show how the LIGO project organized to make steady improvements and ultimately carry out a successful scientific program. He will describe the detections as well as ideas to improve the detectors driven by the new science we hope to learn. Kip will look at the broader aspects of the new field of gravitational wave astronomy. He will tell of the critical role numerical relativity and understanding the quantum mechanics of precision measurements has played. He will also give a vision of the science that could come from an investigation of the gravitational wave sky from periods of fractions of milliseconds to tens of billions of years.

In 1915, a little over 100 years ago, Einstein published the General Theory of Relativity (GR) (Einstein, 1915, 1916a), a new theory of gravitation which replaced the Newtonian force by the idea that mass distorts the geometry of space and the flow of time. Matter then moves in this new space-time along the shortest four-dimensional paths. (Figure 1 and its caption give an impression of the idea.)

The new theory solved a puzzle remaining from Newton's theory for the motion of the planet Mercury, the planet in the strongest gravitational field of the Sun, which after correction for the motions of other objects in the solar system still did not

*The 2017 Nobel Prize for Physics was shared by Rainer Weiss, Barry C. Barish, and Kip S. Thorne. These papers are the text of the address given in conjunction with the award. seem to obey Newton's theory. Specifically, Mercury was not moving along a path dictated by a pure $1 / r^{2}$ force law. To save Newton, another planet between the Sun and Mercury was hypothesized but never found. To Einstein's enormous pleasure, GR gave the measured orbits. It was the first confirmation that he was on the right track. GR also provided some new phenomena open to measurement, small effects difficult to measure but profound in their importance. These were the prediction that clocks ran more slowly in strong gravitational fields than weak ones (Pound and Rebka, 1960; Brault, 1963) and that starlight passing the limb of the Sun would be deflected toward the Sun, the so-called bending of light (Fomalont and Sramek, 1975). It is a tribute to the difficulty of the measurements that both of these effects were only really measured reliably about 50 years later.

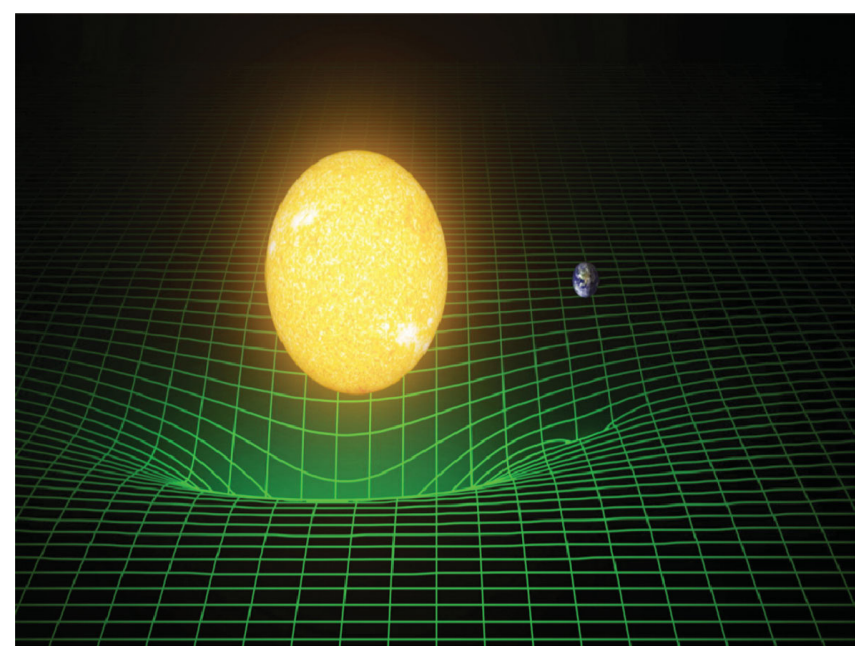

FIG. 1. An attempt at visualizing Einstein's concept of gravity (the General Theory of Relativity) in which distortions in the geometry of space and time have replaced the gravitational force of Newton. Think of a jungle gym of rectangular bars in the three spatial dimensions. The intersection points of the bars are all evenly spaced and straight lines. Imagine also that a clock is placed at all the intersection points and that all the clocks read the same time at the same moment in time. The situation when there are no masses in the vicinity. Now, take a two-dimensional cut through the structure and place the Sun and Earth into the picture. The structure near the Sun and to a smaller extent that near the Earth is distorted. Also, although not shown, the clocks at the intersection points near the Sun and to a lesser extent those near the Earth run a little more slowly than those in the less distorted parts. The orbit of the Earth around the Sun is now dictated by the distortion of space and the time dilation - the path an object with no forces would follow in the new geometry. 


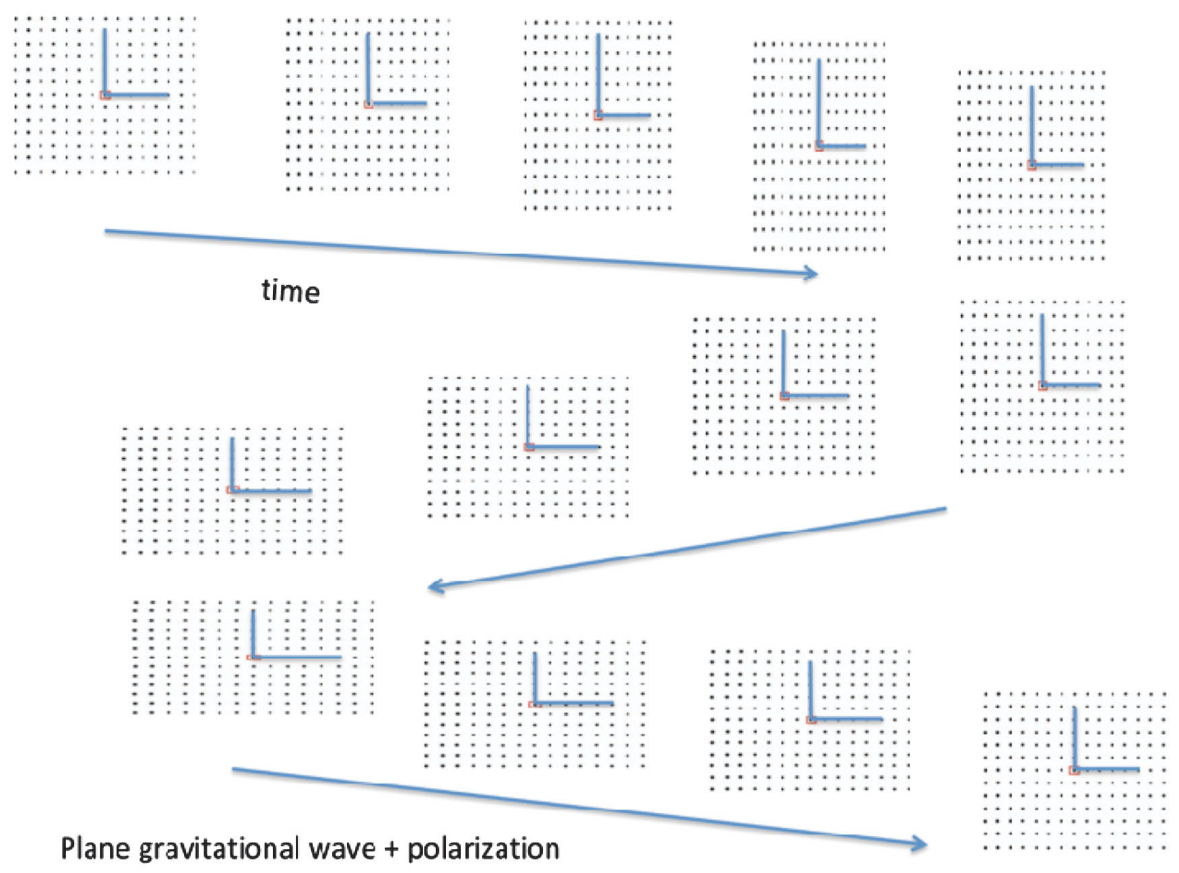

FIG. 2. A gravitational wave + polarization. Gravitational waves are emitted by accelerated masses. They propagate at the speed of light. Once they have become plane waves some number of wavelengths from their source they become transverse waves which cause a strain in space perpendicular to the direction in which they propagate. The strain stretches space in one dimension while simultaneously contracting space in a perpendicular dimension. The strain $h=\Delta l / l$ is the change in distance between two places divided by their separation. The figure tries to show this by distributing a set of probe masses throughout space. The gravitational wave propagates perpendicular to the plane of the figure and the masses. Time evolves from the top left to the bottom right by going through one complete cycle of an oscillating strain. L in the figure symbolizes the LIGO detector. The thing to notice is the change in length of the arms of the $\mathrm{L}$ which becomes the measurable in the detector. The longer the arms the larger is the measurable displacement signal. The extension of one arm with the contraction of the other arm enables the use of a Michelson interferometer which is specifically sensitive to the difference in light travel time along the two arms.

GR had more in it still. In principle, as we have now found directly, one could carry out calculations of massive systems moving at relativistic speeds. Furthermore, gravitational information-gravitational waves-did not travel at infinite speed as implied by Newton's gravity, but traveled at the speed of light as is necessary to be compatible with special relativity.

In 1916 Einstein (Einstein, 1916b) wrote a paper showing some of the ideas discussed above by perturbation calculations using linearized versions of his full field equations. It is in this paper he first describes gravitational waves (Fig. 2).

They travel at the speed of light and are transverse waves much as electromagnetic waves, but rather than exerting forces on charges, they distort space perpendicular to the direction along which they propagate, alternately stretching space in the east-west direction while simultaneously compressing space in the north-south direction. The distortion has the special property that the change in separation of two points is proportional to their separation, the strain $h=\Delta l / l$ in the east-west direction is the same over the entire wave front, while strain is equal but opposite in the north-south direction. [For completeness it is necessary to at least say there is another polarization for the waves rotated by $45 \mathrm{deg}$ to the east-west, north-south directions traveling along the same direction which acts independently (is orthogonal).] The gravitational waves carry energy as well as linear and angular momentum. In the 1916 paper, Einstein describes the wave kinematics well but runs into trouble showing how accelerated masses radiate gravitational waves. In a second paper dedicated entirely to gravitational waves Einstein (1918) derives the quadrupole formula (to within a factor of 2) relating the gravitational wave energy radiated by a mass distribution to its nonspherically symmetric accelerations. Despite the mistake in the 1916 paper, he makes a bold (and for 1916 correct) claim at the end of this paper that gravitational waves will never play a significant role in physics. I have asked the editors of the Einstein Papers Project to find evidence in his notebooks and back of envelopes for the estimates that led him to this, but nothing has been found.

I will be presumptive and guess at what he might have considered, given the technology and knowledge of astronomy open to him in 1916. There are two equations that will help us with this: an estimate for the gravitational wave strain that comes from the quadrupole formula and the relation between the power carried by the gravitational waves and the time dependence of the strain. A way of estimating the gravitational wave strain from the motion of the masses at the source of the waves is

$$
h=\left(\frac{G m}{R c^{2}}\right)\left(\frac{v^{2}}{c^{2}}\right) .
$$

Here $G$ is the Newtonian gravitational constant, $m$ is the mass of the radiating system, $R$ is the distance from the source, $c$ is the velocity of light and $v$ is a nonspherically symmetric velocity of the radiating mass distribution. For example, if the system is composed of two orbiting objects, it becomes the 
relative tangential velocity in their orbits. The term in the first pair of brackets has a special meaning in general relativity, it is dimensionless and is a measure of the absolute strength of gravity in the situation. We who are standing on the surface of the Earth experience the strength of this term as $10^{-10}$, at the surface of the Sun it is about $10^{-6}$, or at the surface of a neutron star it is $10^{-1}$, at the event horizon of a black hole, it is about 1 . It is clear we live in very weak gravity.

The other relation we will find useful is the power per area carried away by the gravitational wave knowing the gravitational strain in the wave

$S_{g}=\frac{c^{3}}{16 \pi G}\left\langle\left(\frac{d h}{d t}\right)^{2}\right\rangle, \quad$ where $\frac{c^{3}}{16 \pi G}=7.8 \times 10^{36} \mathrm{erg} \mathrm{s} / \mathrm{cm}^{2}$.

The power per area in the wave is proportional to the square of the rate of change of the strain times a gigantic factor which tells that a small amount of strain in space is accompanied by a huge amount of energy. In other words, it takes enormous amounts of energy to distort space. One way to say it is, the stiffness (Young's modulus) of space at a distortion frequency of $100 \mathrm{~Hz}$ is $10^{20}$ larger than steel.

An example one might have expected Einstein to use is the gravitational radiation emitted by the collision of two trains, a man-made event of significant energy transfer. Using parameters such as $m=10^{5} \mathrm{~kg}, v=100 \mathrm{~km} / \mathrm{h}$, collision times of $1 / 3$ second and a distance of $R=300 \mathrm{~km}$ (far enough away to be dominated by the radiation rather than Newtonian interaction), one gets a strain $h=10^{-42}$ truly too small to have any physical effect. Another possibility might have been to estimate the change one might be able to detect in observing a binary star system through a telescope. The two stars orbiting each other would lead to the loss of energy by gravitational radiation and would be changing their period as they fall toward each other. Some typical numbers that might have been available in 1916, the two stars both have a solar mass and an orbital period of a day. The energy lost to gravitational waves each orbit relative to the kinetic energy stored in the orbit is about $10^{-15}$ so that it would take about $10^{13}$ years to see the orbit collapse, not a practical astronomical observation.

As with many of the other effects predicted by general relativity, it took the development of technology as well as the improved knowledge of the universe through improved technology to make reliable measurements and observations.

It took until the mid-1970s with discovery of pulsars and vastly improved time keeping to perform the measurements of a binary star system which showed evidence for energy loss due to the radiation of gravitational waves. The definitive measurements were made after the discovery of a binary neutron star system by Russell Hulse and Joseph Taylor (Fig. 3).

Hulse and Taylor tracked the pulsations from a neutron star using the radio telescope at Arecibo (Puerto Rico) beginning in 1972. The frequency of the pulsar was nominally $17 \mathrm{~Hz}$ but they noticed that pulsation frequency was frequency modulated with a period of $8 \mathrm{~h}$. After a considerable effort to establish that there were no other stars in the vicinity, they came to a model of a pair of neutron stars orbiting each other. They established quickly that the dimensionless gravitational field strength of one neutron star at the position of the other was about $10^{-6}$ and that they had come upon a wonderful laboratory to test general relativity. The general relativistic periastron advance was close to $4 \mathrm{deg}$ per year, enormous compared to the $43 \mathrm{~s}$ of arc per

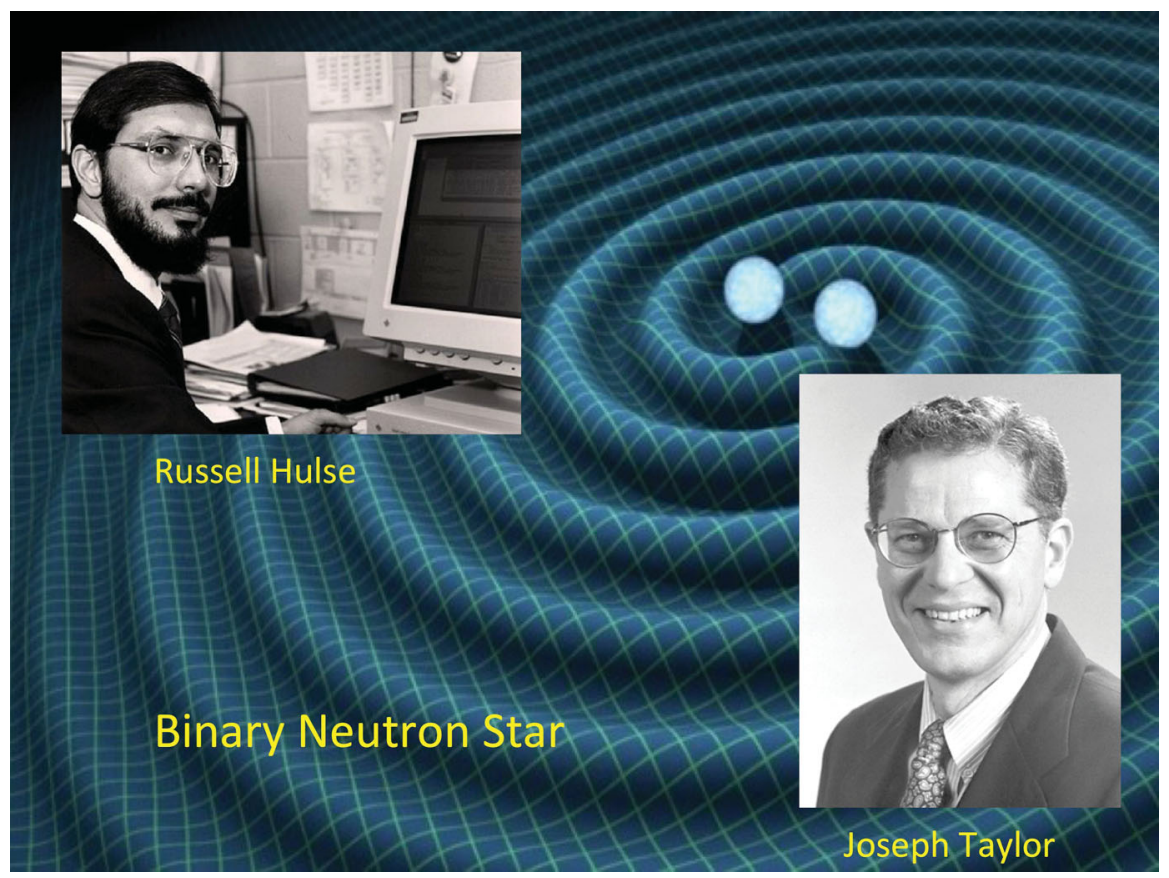

FIG. 3. Russell Hulse and Joseph Taylor were awarded the Nobel Prize in Physics in 1993 for the discovery of the binary pulsar system, which became a remarkable test laboratory for general relativity. They were able to measure relativistic periastron precession of $4 \mathrm{deg} / \mathrm{yr}$ and many other relativistic dynamical effects which allowed them to solve for the individual masses of the neutron stars. As shown in Fig. 4 they solved for the dynamics of this system and minimized the residual between their data and the full relativistic model of the motion; to do this it was necessary to include the loss of energy by the system to gravitational radiation. 
Neutron Binary System - Hulse \& Taylor

PSR $1913+16$-- Timing of pulsars

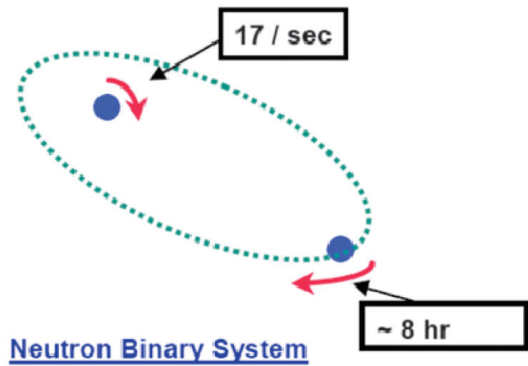

- separated by $10^{6}$ miles

- $\mathrm{m}_{1}=1.4 \mathrm{~m}_{\odot} ; \mathrm{m}_{2}=1.36 \mathrm{~m}_{\odot} ; \varepsilon=0.617$

Prediction from general relativity

- spiral in by $3 \mathrm{~mm} /$ orbit

- rate of change orbital period
Emission of gravitational waves

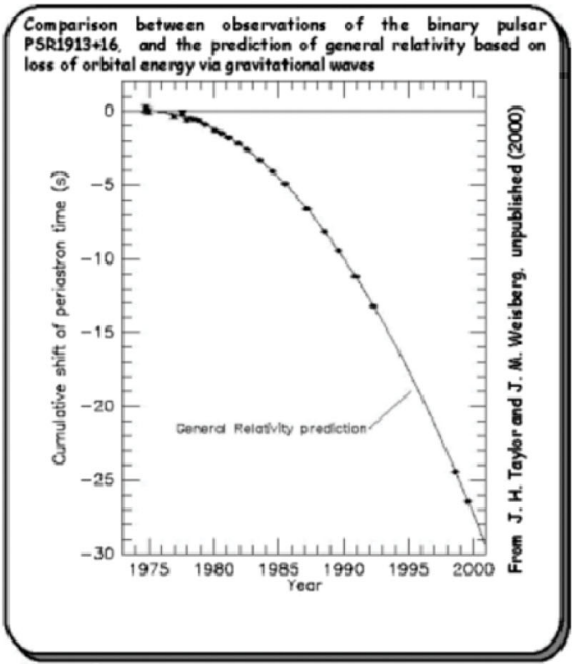

FIG. 4. The change in orbital period of the binary pulsar as a function of epoch. The dots indicate the measured reduction of the orbital period as the neutron stars lose total energy by radiation of gravitational waves. The line is the expectation from the General Theory of Relativity. The system was the first to show the effects of gravitational radiation and also provided the first demonstrated source of gravitational waves for direct detection. As Barry will show, the coalescence of a pair of neutron stars has been detected by LIGO and VIRGO. From Taylor and Weisberg, 1982.

century in the Mercury-Sun system, and through other relativistic terms it was possible to solve for the mass of the two objects separately. One of the more stunning measurements was the change of the orbital period as a function of epoch. Figure 4 shows the period was getting shorter systematically and was consistent with being due to energy being lost by the system to gravitational waves using the quadrupole formula. The explorations of gravitation with this really remarkable laboratory led to Hulse and Taylor receiving the 1993 Nobel Prize in Physics. The discovery not only confirmed the existence of gravitational waves but also identified a source for the eventual direct detection of gravitational waves.

The first experiments that attempted to directly detect gravitational waves from astrophysical sources were an outcome of the Chapel Hill Conference on Gravitation in 1957 (Rickles and DeWitt, 1957). At this conference John Wheeler and Joseph Weber presented the idea of writing the gravitational wave as a tidal force transverse to the propagation direction and treating the detection of weak gravitational waves as a Newtonian interaction of these forces exciting a mechanical resonator. The idea was to measure a gravitational wave arising from an astrophysical event such as a supernova explosion in our own or a neighboring galaxy. The event involved the conversion of a decent fraction of the rest mass of the exploding star into gravitational waves. The frequencies of the motion at the star are in the low $\mathrm{kHz}$ band. The waves, when incident on an aluminum bar with longitudinal resonances in the same band, would drive the bar into oscillation (Weber, 1960). The bar would ring on after the wave had passed through, making it easier to measure.

Figure 5 shows Weber mounting strain gauges on one of these detectors. A critical idea from the beginning was to look for coincident excitations in several bars as a means of making a detection over the noise in the device which was dominated by Brownian motion (the thermally induced random motions of the bar's longitudinal modes). All through the mid- to late 1960s, Weber kept improving the experiment. In 1969 with a detector at the University of Maryland, another at a golf course about 8 miles from the university and a remote detector at the Argonne Laboratory in Chicago, he wrote a discovery paper in

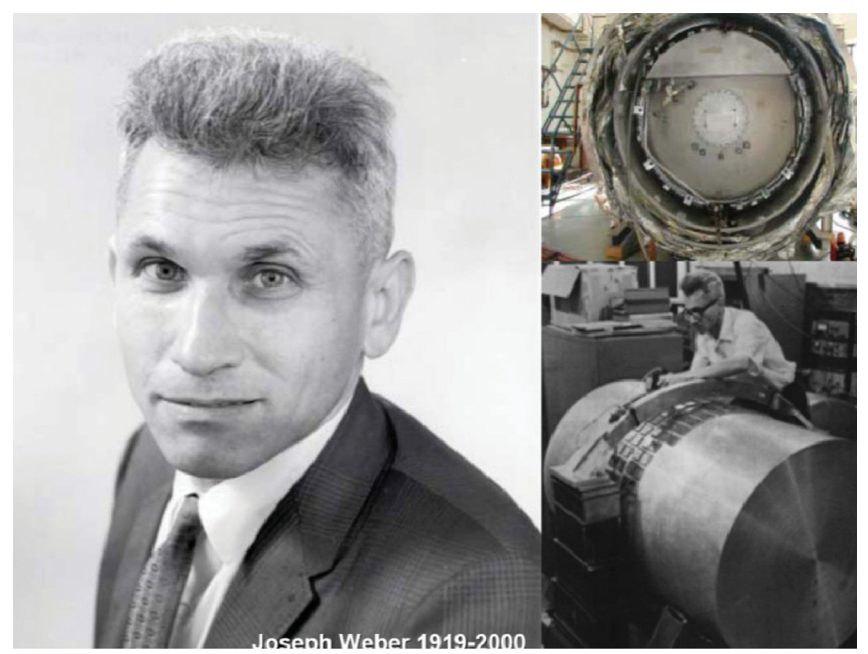

FIG. 5. Joseph Weber was the first to attempt to detect gravitational waves from astrophysical sources directly. The concept for doing this was generated by John Wheeler and Weber at the 1957 Chapel Hill conference on gravitation. The idea was to measure the distortions induced by a gravitational wave in a cylinder. Instead of thinking of the gravitational wave as a strain in space, they thought of the wave as exerting tidal forces on the cylinder and calculated the motion of the bar as an oscillator in inertial space. The tidal force was just another Newtonian force on the bar. Weber imagined short pulses of gravitational waves with some Fourier components at the resonant frequency of the cylinder. The wave would pass through the cylinder and leave it ringing. In the picture on the right, Weber is attaching some strain gauges to the cylinder to measure the excitation. The cylinder is placed in the vacuum chamber (behind him) when searching for gravitational waves. 
Physical Review Letters (Weber, 1969) showing a few coincident pulses per day at a peak strain level of $h \sim 10^{-15}$ in the $\mathrm{kHz}$ band. It soon became evident that if the pulses were gravitational waves from the region around the center of our galaxy, this implied an extremely efficient conversion of rest mass to gravitational waves, in fact the conversion of all the rest mass in the galaxy into gravitational waves in a few million years. Despite this unlikely possibility, about a dozen experimental groups throughout the world began the development of Weberlike detectors. By the early 1970s it was becoming clear that no one was able to confirm the Weber measurements.

In 1966 I was asked by the MIT Physics department to teach a course in General Relativity. It was at the time of the revived interest in GR in the physics community which had begun with the Chapel Hill meeting and with the increased research in experimental work in gravitation due to Dicke and Wheeler at Princeton and Schiff at Stanford and others. I had just returned to MIT from a postdoctoral position with Dicke and had started a new group in Cosmology and Gravitation. For the prior 40 years GR had not been taught in physics at MIT but had occasionally been taught in the mathematics department as part of differential geometry. What little I knew of the physics of GR had come from conversations with colleagues at Princeton and simply from reading. The formal mathematics of differential geometry and tensor analysis was entirely new to me and at the time there were not yet really good textbooks on the subject. It was a hard term, often learning from the students and just catching up to them. The students in the course were aware of the Weber experiments and asked for lectures about them. By that time in the course, I had become a convert to Einstein's geometric view and had a hard time looking at gravitational waves as a tidal force and, especially, the idea of a metal bar interacting with the wave.

I spent the weekend before the lecture trying to apply F. A. E. Pirani's (Pirani, 1956) (Fig. 6) approach of the

\section{Michelson Interferometer Schematic and GW sidebands}

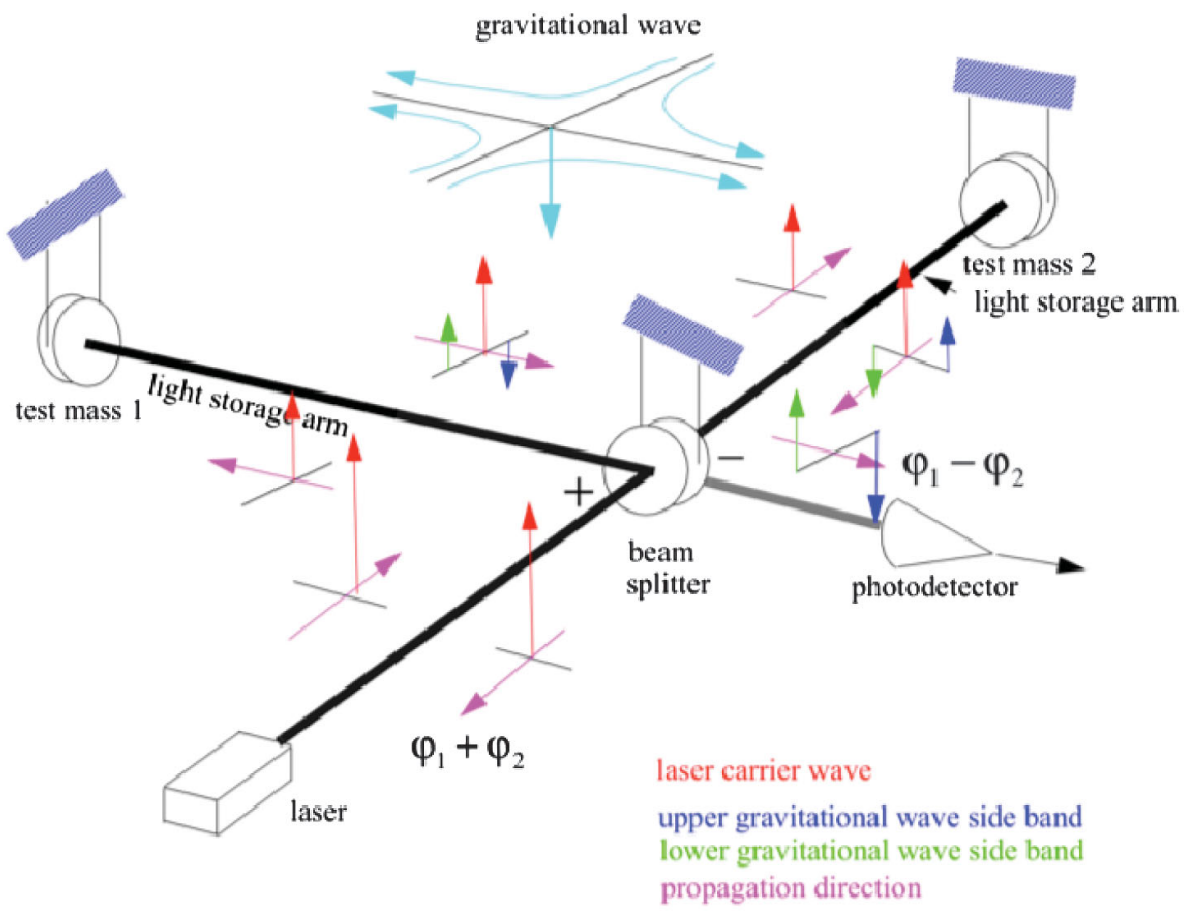

FIG. 6. Schematic of a Michelson interferometer used as a gravitational wave detector. The gravitational wave at a low frequency $f_{g}$ is incident from above the plane of the interferometer. Start by following the light from the laser to the symmetric port of the beam splitter ( + no phase inversion of the light on reflection). The laser generates carrier light (red amplitude). The violet vectors indicate the propagation direction of the light. The laser light that gets reflected by the beam splitter heads to the mirror on test mass 1 reduced by the reflectivity of the beam splitter. On reflection from test mass 1 , which is moving due to the gravitational wave, the carrier generates two sidebands one at a frequency $f_{g}$ above the carrier (blue) and another $f_{g}$ below (green). The beam heads back to the beam splitter. The beam from the laser that gets transmitted by the beam splitter heads toward test mass 2; the carrier is reduced by the transmission of the beam splitter. That beam on reflection from test mass 2 also gets two sidebands from the motion of the test mass 2 , but they have the opposite sign because the gravitational wave is compressing space on the test mass 1 side while expanding space on the test mass 2 side. The beam from test mass 2 gets reflected toward the detector from the antisymmetric port of the beam splitter (the phases of the carrier and the sidebands are inverted). The beam from test mass 1 is transmitted by the beam splitter toward the detector. The beams from the two sides of the interferometer are added. If the paths on the two sides take equal time from the first encounter with the beam splitter to the second one (or take a difference in time that is an integral number of periods of the light wave), the carrier cancels while the sidebands double at the detector. The sidebands carry the information about the gravitational wave to both the wave amplitude and the phase. To make the sidebands detectable as a current in the photodetector requires a small amount of carrier to beat against the sidebands. 
geodesic deviation to a simple gedanken experiment to measure a gravitational wave. Eventually it turned into a straightforward idea of measuring the time it took light to travel back and forth between two free masses. With a light source mounted on one of the free masses, one set a clock also mounted on the mass to start as the light left to go to the distant mass. At the distant mass the light was reflected by a mirror back to the source and the clock was stopped by the arrival of the return light. One did this measurement when no gravitational wave passed between the two masses and when a wave did pass and compared the times. It turns out an easy problem to set up in GR and since all the measurements were made at the same mass there was no question of the conversion of coordinate time to proper time. After this we went on to cosmology and more interesting problems. Unknown to me two Russians, Gertsenshtein and Pustovoit (Gertsenshtein and Pustovoit, 1963), had come up with a similar idea.

By early in the 1970s, when it became evident that there were no confirmations of the Weber experiments, I returned to thinking whether it was actually possible to convert the gedanken experiment in the GR course to a real experiment (Fig. 6). The real experiment would use a Michelson interferometer configuration to exploit the symmetry of the gravitational waves.

Initial interferometric GW detector groups late 1970's

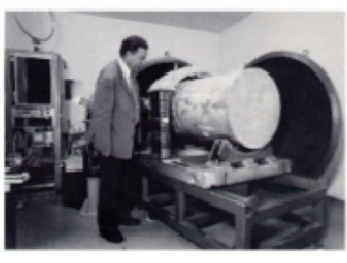

$H$. Billing

Max Planck Garching

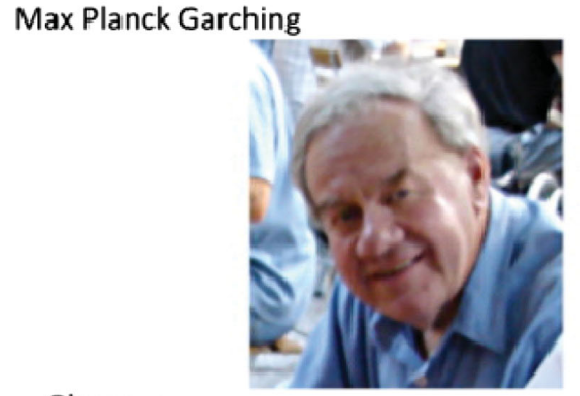

Glasgow

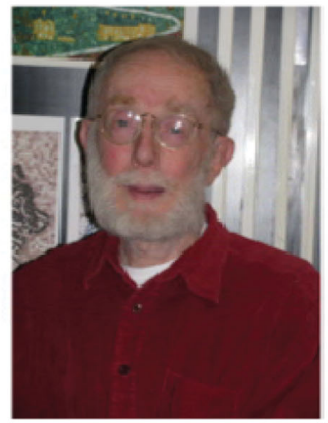

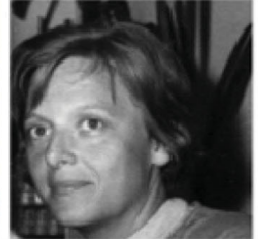

L. Schnupp

R. Drever

F.A.E. Pirani

MIT

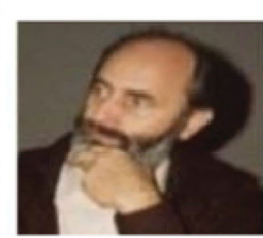

K. Maischberger

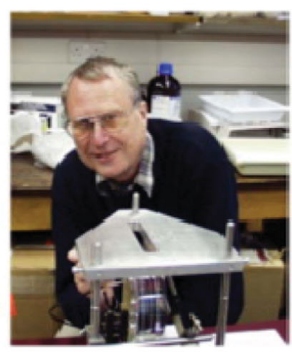

J. Hough

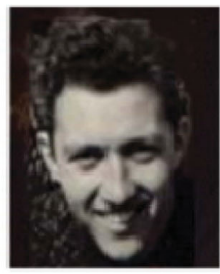

W.Winkler

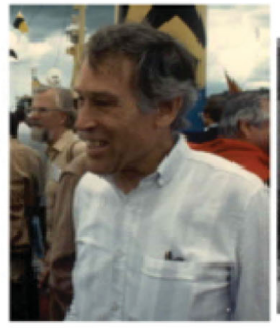

R. Schilling

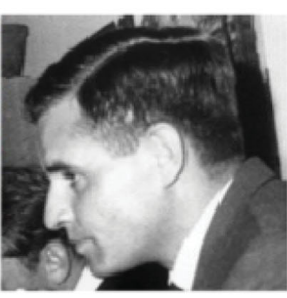

A. Rudiger

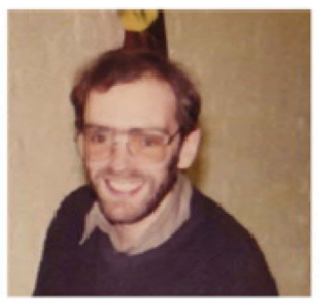

B. Meers

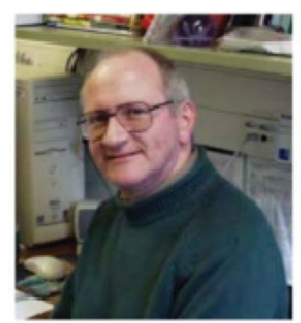

H. Ward

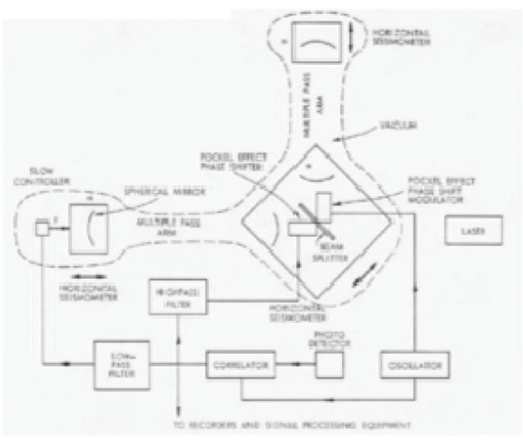

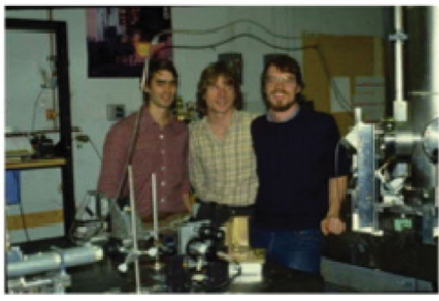

J. Livas, D.H. Shoemaker, D. Dewey

FIG. 7. Bottom left: F. A. E. Pirani, who showed that it is possible to measure the relative motion of two free masses traveling through a gravitational wave (so-called geodesic deviation) in a coordinate-independent manner, a critical idea that was not fully accepted until the 1957 Chapel Hill Conference. Bottom middle: A schematic diagram of an interferometric gravitational wave detector with suspended masses, multiple passes along the interferometer arms and a technique to reduce the amplitude noise of the laser light. Bottom right: The 1.5-meter prototype at MIT and the three graduate students who constructed and operated it. Top line: The gravity research group at the Max Planck Institute of Astrophysics in Garching, Germany, who built a 3-meter interferometer prototype and then a 30-meter one to show that the idea scaled properly. The group invented many of the solutions for the noise in these systems including the idea of suspending all the optics in the phase sensitive part of the interferometer and the need to frequency stabilize the laser to deal with scattering by stray light. Schnupp came up with the idea to make a slight imbalance in the interferometer arms to use external phase modulation. Schilling invented the idea of power recycling to increase the modulated light in the interferometer. Middle line: The gravity research group in Glasgow, Scotland, Drever developed Fabry-Perot cavities as multipass elements in the interferometer arms and also invented the idea of power recycling (Drever, 1983). Brian Meers (Meers, 1988) was the first to suggest putting another partially reflecting mirror between the photodetector and the beam splitter to tailor the interferometer spectral response by reflecting the sidebands back into the main interferometer arms. Ward developed a method to align the interferometer. 

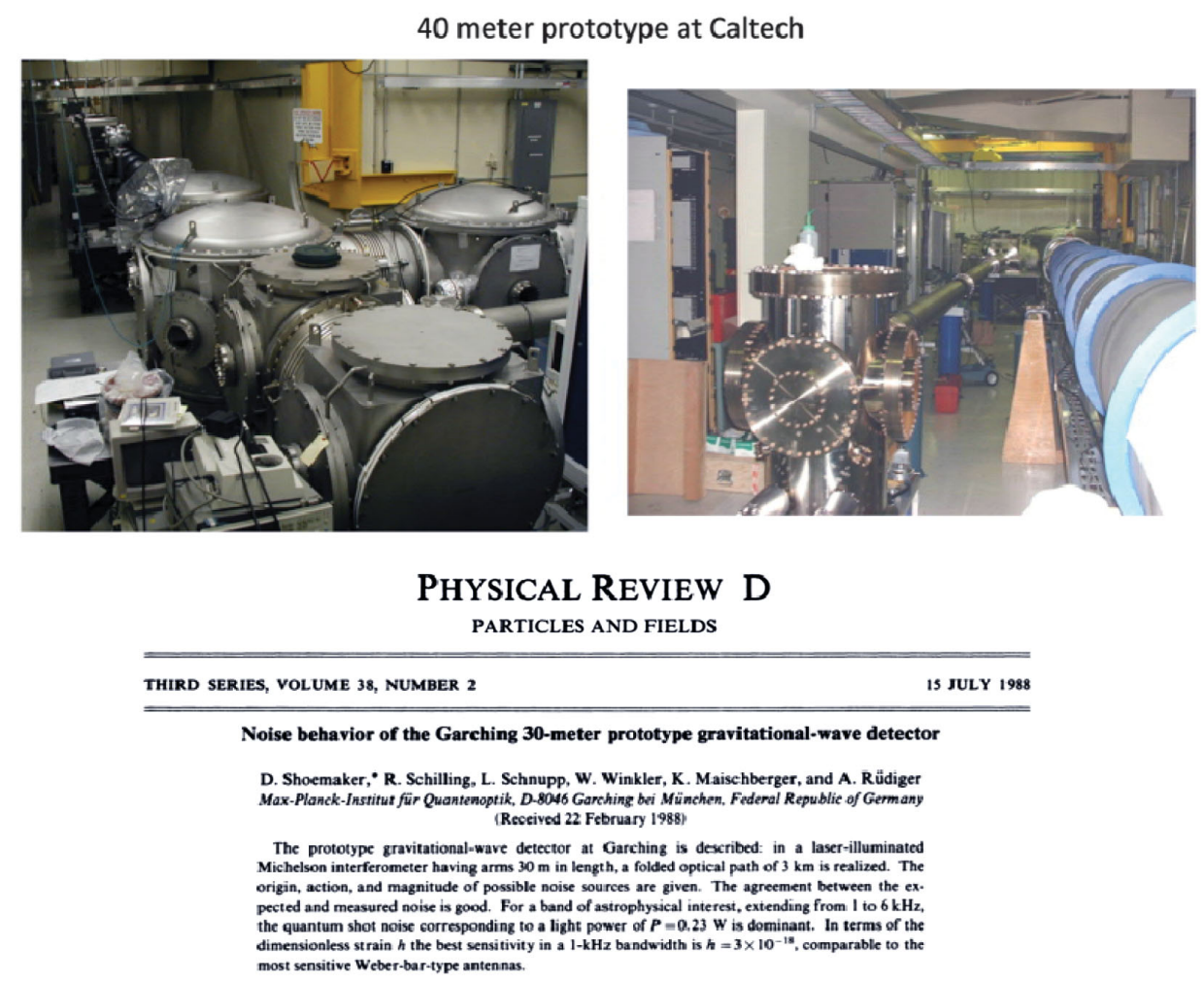

FIG. 8. The abstract of the paper on the 30-meter interferometer operated by the Max Planck Group in Garching showing that an interferometer had attained better sensitivity than the best cryogenic Weber bar detectors. The 30-meter results were important in helping to make the case for long baseline instruments such as LIGO. The top two photos are of the 40-meter instrument constructed at Caltech by Whitcomb and Drever. The 40-meter at Caltech and the 5-meter at MIT (Fig. 13) were the final test beds for the first LIGO detector.

The interferometer would need some unusual properties: the mirror masses were suspended so they could move in response to the wave, the light was to be reflected back and forth many times along the interferometer arms, and to get to the shot noise limit (quantum limit) one needed a method to high frequency modulate the light above the excess noise of the available laser sources (Fig. 7). A study of the various fundamental physics noises in such an instrument and of the environmental noise sources showed by making the instrument on $\mathrm{km}$ scales there was a chance that one could intersect the strain sensitivity required for detection of some astrophysical sources. I put the calculations in a Quarterly Progress Report of the Research Laboratory for Electronics (Weiss, 1972) and asked the Laboratory management to help fund the construction of a 1.5-meter prototype using military research funds (Fig. 7).

At that time in the early 1970s the primary condition required by the military to support research was to train scientists and engineers. By 1974 one of the corrosive effects of the Vietnam War was the demand by many of the antiwar demonstrators to starve the war effort by insisting the military only support research essential for their mission. This was also urged by the supporters of the war in the administration who distrusted academic scientists in general. As judged by the Director of the Research Laboratory of Electronics, cosmology and gravitation research was not relevant to the military's mission and the lab support was terminated. I then began a multiyear effort to gain support from other federal agencies but ran up against a skepticism among peer reviewers of whether interferometric methods using free masses could be used to detect gravitational waves as well as increased pessimism about gravitational wave research in general.

Scientists in Europe who had been involved in attempting to confirm the Weber results had become interested in the interferometric free mass detector. The group at the Max Planck Institute of Astrophysics in Garching led by Heinz Billing had collaborated with an Italian group in Frascati to run coincident bar detectors. They had done a thorough analysis of the noise in their detectors and designed a data

The measurement challenge

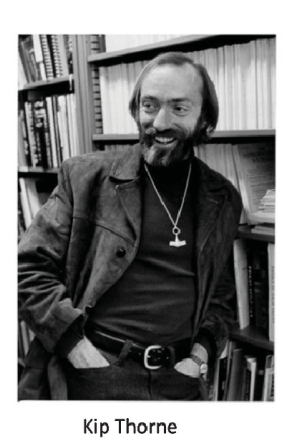

$$
\begin{aligned}
& \mathrm{h}=\frac{\Delta \mathrm{L}}{\mathrm{L}} \leq 10^{-21} \\
& \mathrm{~L}=4 \mathrm{~km} \quad \Delta \mathrm{L} \leq 4 \times 10^{-18} \text { meters }
\end{aligned}
$$

$\Delta \mathrm{L} \sim 10^{-12}$ wavelength of light

$\Delta \mathrm{L} \sim 10^{-12}$ vibrations at earth's surface

FIG. 9. Kip Thorne around 1980 with his measurement challenge to the experimenters based on his and others' estimates of possible astronomical gravitational wave source dynamics and populations. 
acquisition system with adequate bandwidth to fully characterize their signals and also had developed software algorithms that provided meaningful false alarm probabilities. They saw no coincident pulses. They were deciding whether to drop out or develop cryogenic detectors or to try free mass interferometers. They decided to build a 3-meter interferometric prototype and quickly established the fundamental noise processes that would limit performance, some of which I had estimated in my study but also others which I had neglected (Fig. 7). They worked on this prototype systematically diagnosing the noise and designing methods and concepts to circumvent the noise. Eventually they were limited by the fundamental quantum and thermal noise. At that point in their research they designed and constructed a 30-meter prototype (Shoemaker et al., 1988) to test the scaling laws toward km scale instruments (Fig. 8).

Another group in Glasgow, Scotland, led by Ronald Drever (Fig. 7) also became interested in interferometric free mass detectors after having worked with bar detectors. The experience here was a little different. They had redesigned the bar detector to be broadband and were now more disturbed by noise generated in the motion transducer. They became interested in the possibility of using an interferometric rather than a piezoelectric sensor as readout. They also eventually went to a free mass design but decided on optical cavities (Fabry-Perot interferometers) as the means of bouncing the light back and forth in the interferometers arms instead of the Herriot delay lines (discrete spots on the mirrors) I had proposed. They came to this design by noting that delay lines were more prone to phase noise from optical scattering than a Fabry-Perot.

An important turning point occurred in 1975 when Kip Thorne (Fig. 9) and I met in Washington, D.C. to work on a committee to study the possible role of the space program in research on gravitation and cosmology. At the time Kip had established one of the premier groups in theoretical gravitation at Caltech and was thinking of encouraging Caltech to start a new complementary program in experimental gravitation. The issue we discussed was the nature of such a program. Kip had been thinking of gravitational wave research and had come to realize that a program ultimately going to be able to measure gravitational waves from "allowed" astrophysical sources would require sensitivities $h \leq 10^{-21}$ in the 10 to $1000 \mathrm{~Hz}$ band, a million times more sensitive than the Weber bars. Kip had been much influenced by Vladimir Braginsky (Moscow State University), whose notion was to develop bars operating
A STUDY OF A LOWG BASELINE

GRAVITATIONAL WAVE ANTEMNA SYSTEM
Prepared for the Nationall science Foundation under NSF Grant PHY $=81.09581$

to the Masadehusetts Institute of Technology

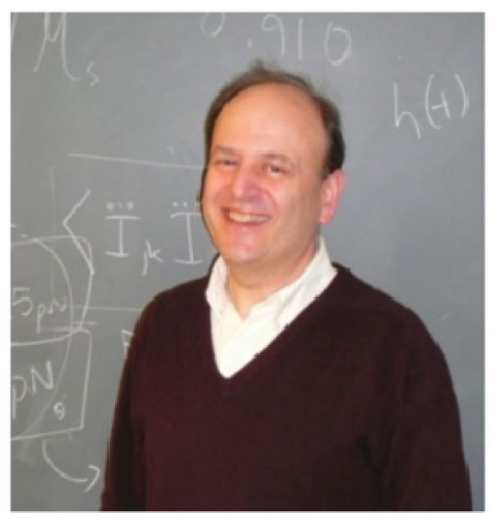

P. Saulson

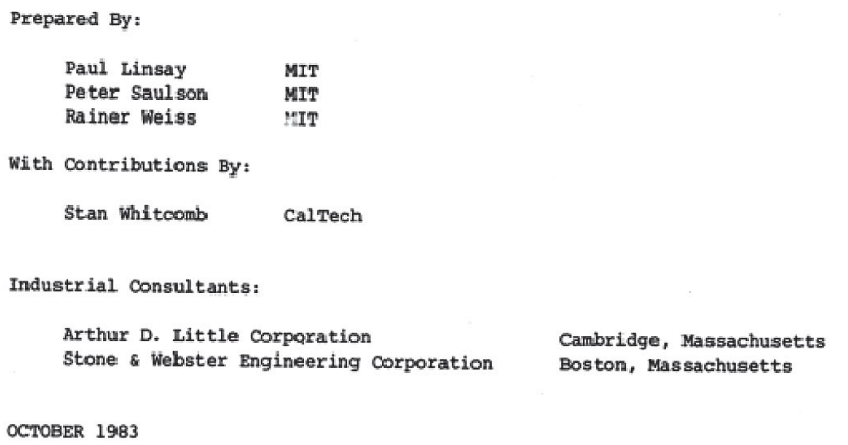

Prepared By

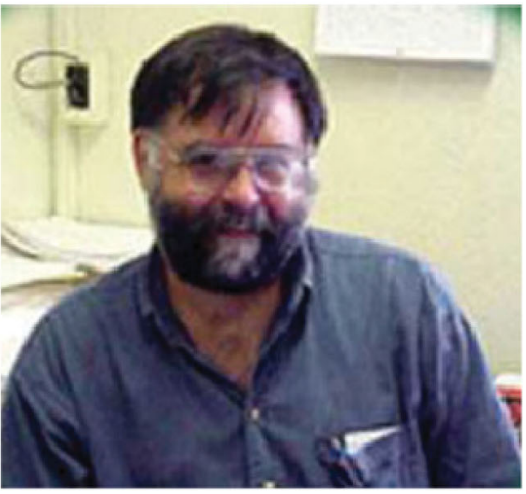

S. Whitcomb

FIG. 10. In 1979 the NSF supported a study (Linsay et al., 1983) with industry to look at the feasibility of building a multikilometer baseline interferometric gravitational wave detector. The study summarized the current state of prototype research and estimates for gravitational wave sources. It looked at the ability of industry combined with academic research to develop adequate optics, lasers, vibration isolation and control systems to meet the scientific requirements. The designs and costs of the vacuum system and the buildings as well as cost scaling relations were developed. Possible sites for up to $10 \mathrm{~km}$ baseline systems both above and below ground were investigated. One of the intents of the study was to be the factual basis for a proposal to the NSF by a consortium of scientists and institutions to build a pair of detectors in the United States. 
near the quantum limit. I suggested he think about long baseline interferometers and look into bringing people from the Max Planck or Glasgow groups to Caltech to start such a program. In 1978 Caltech did make the decision to form a new group with Ronald Drever as its leader and with a significant internal Caltech investment. In 1980, Stan Whitcomb was brought to Caltech to help lead the new Caltech Group.

The combined facts of the success of the Max Planck group in demonstrating the scaling relations for the interferometers, the progress in gaining sensitivity in the Max Planck as well as the MIT prototypes and the significant investment in the field being made by Caltech led me to make a proposal to the NSF to study what was involved in actually making an interferometric detector with adequate sensitivity to detect astrophysical sources. Our study involved several scientists working (Fig. 10) with industrial engineering consultants to look at designs and costs of the vacuum system, the large-scale optics and lasers, and the siting for the construction of two multikilometer interferometric detectors separated by continental distance. The aim of the study was to establish feasibility and cost scaling relations for the large infrastructure and to look at the experimental challenges to gain the two large factors in the detector design itself. With multikilometer long arms measuring a strain of $10^{-21}$ requires a displacement sensitivity of $10^{-18}$ meters, about $1 / 1000$ of the size of a proton. Using light with a wavelength of $10^{-6}$ meters, one needs to develop optical techniques that can measure $10^{-12}$ of a wavelength. Furthermore, typical ground vibrations even in quiet places are broadly around $10^{-6}$ meters, so that to be able to sense a gravitational wave above just ground noise one

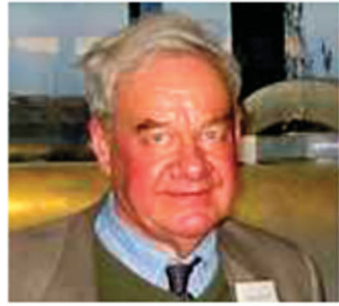

R.Drever

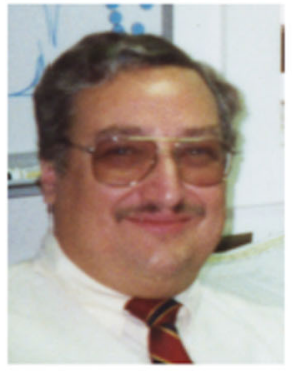

F. Raab

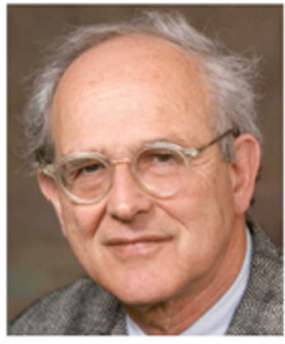

R. Weiss

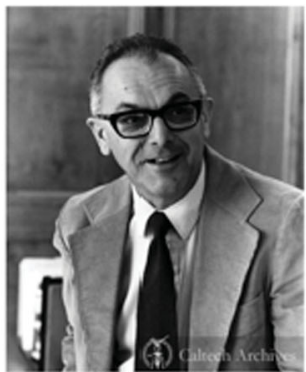

R. Vogt

Proposal to the National Science Foundation

THE CONSTRUCTION, OPERATION, AND SUPPORTING RESEARCH AND DEVELOPMENT OF A

\section{LASER INTERFEROMETER GRAVITATIONAL-WAVE OBSERVATORY}

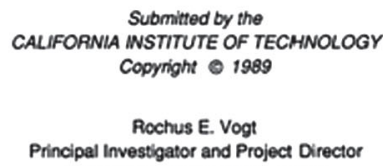

Kip S. Thome

Co-Investigator

Calfornia Institute of Technology

Rainer Weiss

Co-lnvestigator

Massachusetts Institute of Technology

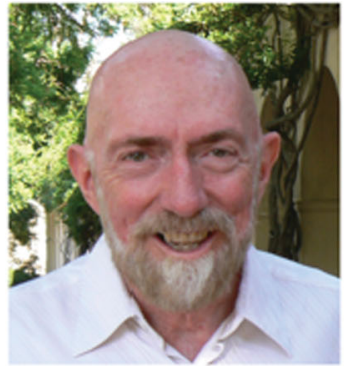

K. Thorne

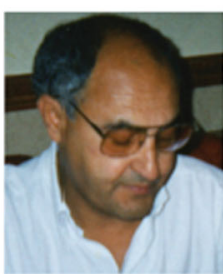

F. Asiri

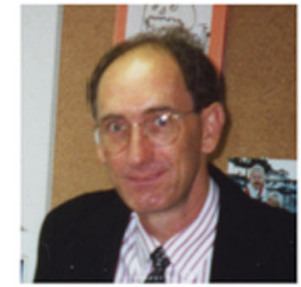

R. Savage

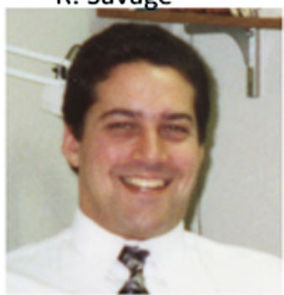

M. Zucker

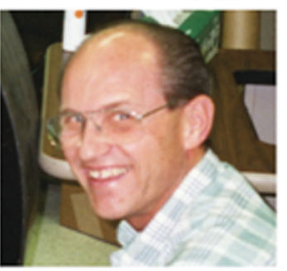

L.Jones

FIG. 11. The 1989 proposal (Vogt et al., 1989) to build and operate LIGO. Rochus (Robbie) Vogt, who had become Director of the LIGO project in 1987, organized a joint Caltech/MIT collaboration to write the proposal. The proposal specified specific parameters for the project: two sites run in coincidence-one in Hanford, Washington and the other in Livingston, Louisiana-a 4 km baseline, an initial detector design and strain sensitivity spectrum, an environmental monitoring system, the vacuum system for the instrumentation and the vacuum system for the beam tubes, the buildings and permanent infrastructure. The proposal also provided a plan for a developmental approach for the detector with an initial detector using then available technology, having the possibility for detection; and a later advanced detector with not yet fully available technology, in the development stages at the various LIGO research laboratories, having a good chance for detection. The facilities were designed to accommodate both the initial and the advanced detector. I worked with engineers Boude Moore and later Larry Jones on the design, construction and qualification of the $4 \mathrm{~km}$ beam tubes. 


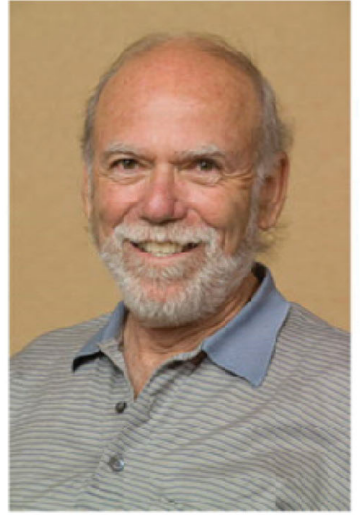

B. Barish

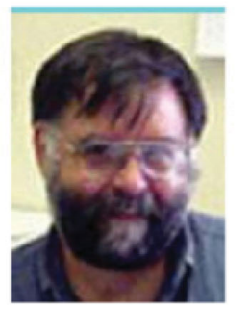

S. Whitcomb

\section{The real start}

1994

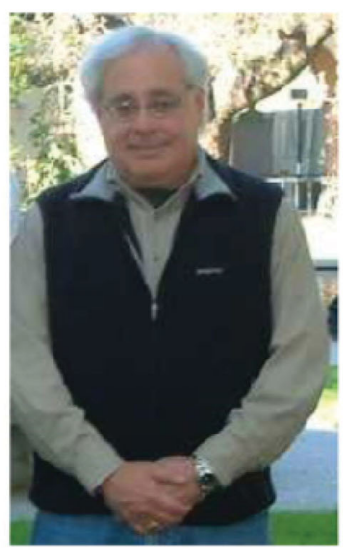

G. Sanders

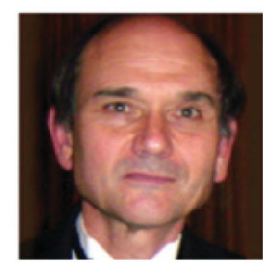

A. Lazzarini

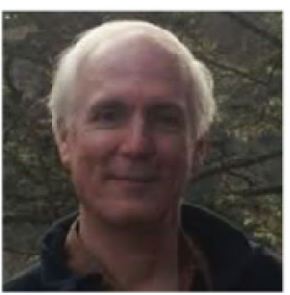

D. Coyne

FIG. 12. By 1994 the NSF as well as both the Caltech and MIT administrations felt the management of the LIGO project needed strengthening. In 1994 Professor Barry Barish of Caltech became Director of LIGO. He substantially increased the management and technical staff of the project. He brought in Dr. Gary Sanders as the Project Manager and engaged Dr. Albert Lazzarini and Dennis Coyne as system scientist and engineer. He asked Dr. Stan Whitcomb to manage the design and construction of the first interferometer. Barish also realized that a larger group than only the Caltech and MIT groups would be needed to analyze the data and to publish results. He was the architect for the LIGO Scientific Collaboration. Barry asked me to be the first spokesperson for the Collaboration.

would need to isolate the mirrors that determine the end points of the interferometer to another factor of $10^{-12}$. Reducing the noise in the system by these two factors of $10^{-12}$ was known at the outset to be the primary challenge. The intent of the study was to serve as the basis for a collaborative proposal by all groups interested in the construction of LIGO.

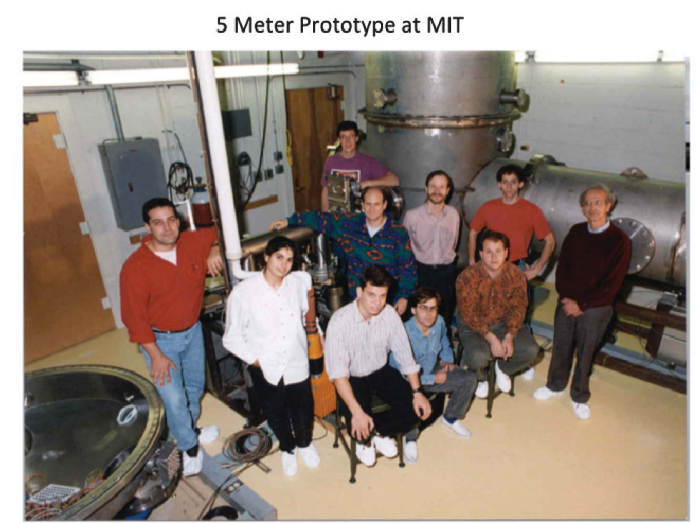

Test of LIGO alignment system

Test of power recycled Michelson-Fabry Perot interferometer @ 0.5 and 1.06 microns Test of suspended power recycled Michelson

Perturbed Optics Modeling

FIG. 13. The MIT group in the 1990s and some of the research that was done. Front row, left to right: Michael Zucker, Nergis Mavalvala, Peter Csatorday, Peter Fritschel, Joseph Kovalik, and R. Weiss. Next row back: Yaron Hefetz, David Shoemaker, and Brett Bochner. Back: Brian Lantz.
The results of the study were presented to an NSF committee considering large new projects in physics in 1983. The presentations were given jointly by the Caltech and MIT research groups who had begun to form a collaboration. The committee was remarkably encouraging in their evaluation:

The committee is impressed with the long-range scientific potential of gravitational wave detection. It will not only test our basic understanding of gravitation, but provide an entirely new window on the universe.

We have considered the major interferometric laser detection system now being developed by the Caltech and MIT groups. We note that not only is this an outstanding scientific opportunity, but the Foundation is the only source of support for ground based gravitational physics.

As with any attempt at a qualitative advance, there are risks: here the uncertainties involve both the magnitude of the signals to be detected and the large extrapolation of known experimental technique inherent in the proposed scale. We find, however, the fundamental scientific merits of such an investigation so important as to be worth a substantial investment (National Science Foundation, Advisory Committee for Physics, December 12-13, 1983). 
Following this encouragement from the NSF committee we began making the transition from independent investigator small-scale science to the project organization required for a large-scale coordinated scientific effort. At first, we did not succeed; it took until 1994 with Barry Barish becoming Director of LIGO to truly make this transition. In the interim there was progress in the technology in the various laboratories but only halting progress in major project decisions such as choosing sites or more than a conceptual design for the interferometer configuration. In 1986 we began an effort to seek significant funding for the interferometric gravitational wave detection program. Richard Garwin suggested that the NSF carry out a summer study of the field by an independent group to both reevaluate its importance as well as to assess readiness and cost. A "Panel on Interferometric Observatories for Gravitational Waves" was established consisting of established scientists with experience in large projects, experts in the various technologies required and scientists with knowledge of the relevant aspects of gravitation and astrophysics. In January 1987 they issued their report, which encouraged the NSF to build two full scale interferometric detectors at widely separated sites and insisted that the project find a single Director before moving forward.

Robbie Vogt became the first Director of LIGO in 1987. He introduced a structure into the laboratory research program assigning responsibilities for both detector development and infrastructure planning and estimation. His major effort was to guide us, both Caltech and MIT participants, scientists and engineers, to write a construction proposal for LIGO with sufficient detail to estimate schedules and costs. The proposal also served to pull together in one place the knowledge of gravitational wave sources, the noise budgets for the detectors and the plans for data analysis to establish confidence of detection. It became a source book for the field (Vogt et al., 1989) (Fig. 11). The proposal also laid out the plans for the field with a staged strategy. The infrastructure would be designed and built to be adequate for both an initial detector using technology we now had (almost) in hand and would not compromise a future advanced detector we could envision operating at the quantum limit of a 1 ton test mass. In the process of writing the proposal, Vogt paired an engineer with a scientist to assure that the planned infrastructure would be able

\section{Advanced LIGO Fabry-Perot Michelson Interferometer Schematic}

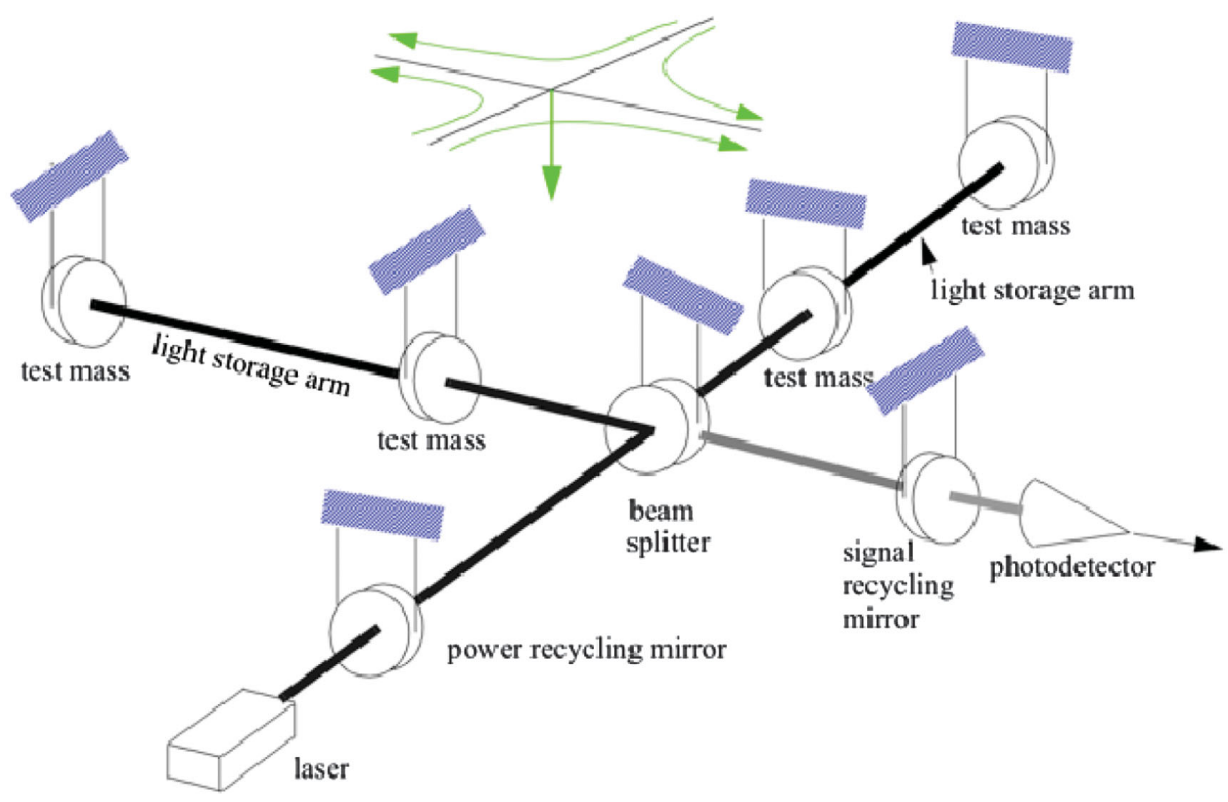

FIG. 14. A schematic diagram of the advanced detector that made the 2015 measurement of the gravitational waves from a binary black hole collision. The interferometer has all the ingredients needed to make the factor of $10^{-12}$ sensitivity improvement in measuring the small displacements of the test masses. To the main elements of the interferometer presented in Fig. 7 are added input test masses with partially transmitting coatings between the beam splitter and the end test masses. The combination of the input and end test mass comprises an optical resonator (Fabry-Perot cavities) which can be thought of as bouncing the light between them many times (typically several hundred times) to increase the gravitational wave induced sidebands by the number of bounces before returning the light to the beam splitter. One attempts to make the number of bounces the same in both arms. As in Fig. 7 the sidebands from the two arms add going toward the photodetector and the carrier cancels. No sidebands return to the laser, but all the carrier does. Between the beam splitter and the laser a new partially reflecting mirror is inserted, the power recycling mirror. The position of this mirror and its transmission is arranged to make another interferometer that cancels the carrier from the laser reflected by the power recycling mirror with the carrier transmitted back by the recycling mirror from the beam splitter. This eliminates the carrier being reflected by the interferometer to the laser and builds up the carrier power between the beam splitter and the input test masses by several hundred. This is the equivalent of using a more powerful laser. The optical configuration described was used in the initial LIGO interferometer. Finally, the advanced detector includes another partially reflecting mirror between the beam splitter and the photodetector, the signal recycling mirror. This mirror reflects the sidebands back into the interferometer and modifies the spectral response of the entire interferometer to the sidebands, thereby tuning the spectral response of the detector to the gravitational waves being sought. 


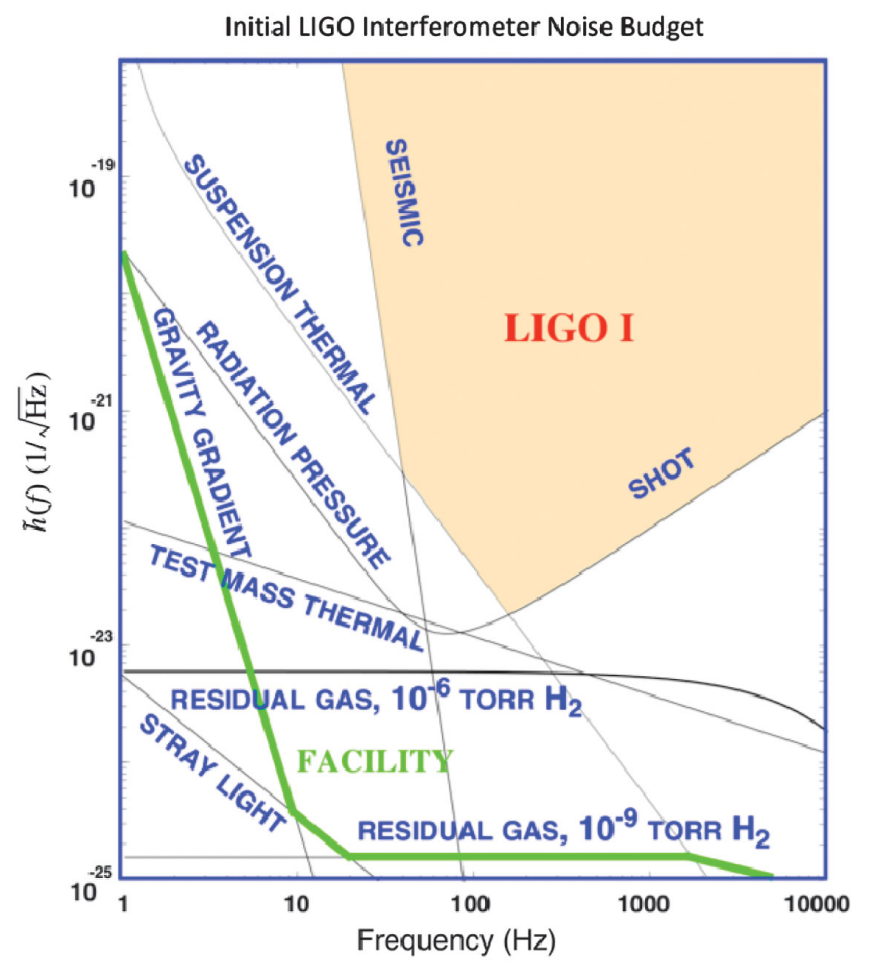

FIG. 15. The noise budget of the initial LIGO interferometer as described in the 1989 proposal. Note the vertical axis is the spectral amplitude strain $/ \sqrt{\mathrm{Hz}}$. To convert to the rms strain, which has been used in other parts of this paper, multiply the plotted value by the square root of the detection bandwidth. At approximately $100 \mathrm{~Hz}$ with a $100 \mathrm{~Hz}$ detection bandwidth, the rms detector sensitivity is about $10^{-22}$ strain. The plot shows the principal noise terms that limit the performance of the detector. At frequencies above $200 \mathrm{~Hz}$, the quantum noise (shot noise) limits the performance. The line labeled radiation pressure, which does not limit this detector, is the other part of the quantum noise due to the fluctuations in radiation pressure on the mirror. The two terms together constitute the quantum limit and are the equivalent of the noise in a Heisenberg microscope but here with a macroscopic object such as a $10 \mathrm{~kg}$ mirror rather than an electron. In this naive rendering one can reduce the shot noise by increasing the optical power but at the expense of increasing the radiation pressure noise. The noise near $100 \mathrm{~Hz}$, the sweet spot for this detector, is limited by the thermal noise in the pendulum suspension, in this case phonon excitations at the end points of the pendulum. At frequencies below $70 \mathrm{~Hz}$ the detector is limited by incomplete removal of the ground vibrations (seismic noise) at the mirror. Seismic noise and pendulum thermal noise were greatly reduced for the advanced detector (Fig. 16). The broad noise spectra called residual gas are the phase fluctuations induced by forward scattering of residual gas molecules traveling through the optical beams in the long arms. This term is the primary reason for ultrahigh vacuum in the beam path. The sharply rising curve at low frequencies labeled gravity gradients is due to the fluctuating Newtonian gravitational forces on the end test masses caused by time dependent density fluctuations in both the atmosphere and the ground. Even though there is some hope in being able to measure these fluctuations or reduce them by burying the instrument, these constitute a severe limit to measure low frequency, less than a few $\mathrm{Hz}$, gravitational waves on the ground. to serve the experiment requirements. Vogt also oversaw the site selection.

After a stormy period in the project, Barry Barish became the second Director of LIGO in 1994. He instituted significant changes in the project organization by broadening the leadership from a skunk works to a larger managerial group (Fig. 12). He also conceived and organized the LIGO Scientific Collaboration, which included both LIGO project members as well as groups outside of Caltech and MIT who were interested in the new science to come from LIGO. The Collaboration has major roles in the data analysis and publications of the science. Some groups in the Collaboration are involved in basic detector research as well.

By the mid-1980s the technical development to accomplish the two factors of $10^{-12}$ was advancing in several research laboratories: a 40-meter prototype at Caltech, a 5-meter prototype at MIT (Fig. 13), a 10-meter prototype in Glasgow at a new laboratory in Hannover where the Max Planck group had moved from Garching, and a new program joint between Italy and France in Cascina, Italy (VIRGO). Instead of continuing with the historical development I will turn to the major ideas to gain the two factors.

The optical arrangement of the advanced detector, for which Barry will show some of the results, is schematized in Fig. 14. The initial LIGO detector was the same, except there was no signal recycling mirror. The caption explains some of the basic concepts. The noise budget for the initial long baseline detector in the LIGO facilities is shown in Fig. 15, giving both the estimated contributions from fundamental physical principles and contributions from technical noise that can be reduced by further engineering. The noise budget is an excellent way to keep track of our progress in accomplishing the two factors of $10^{-12}$, and, as Barry will show with real data from the instrument, the slow but steady progress made as we learned more about the instrument in the commissioning.

A significant step in achieving the improvement against seismic and pendulum thermal noise was made in the development and construction of the advanced detector, Fig. 16. The two major improvements were a new suspension system with four pendula in series each stage providing a filter against seismic displacement noise varying as $1 /$ frequency $^{2}$. The final stage supporting the test mass using very low mechanical loss fused silica fibers (rather than steel fibers) to reduce the suspension thermal noise. The suspension system was provided by the Glasgow group. The second improvement was a multistage active vibration isolation system to further reduce the seismic motion, especially at low frequencies where the pendula are not effective. The results of these developments will be described by Barry in the next talk.

I started this talk with an acknowledgment of the remarkable role the National Science Foundation played in fostering and supporting LIGO over the 45 years from an idea to the first detections and the opening of a new field of science. All the more remarkable as it was risky science and the NSF saw the project through significant external criticism and our internal disorganization. Much of the responsibility and foresight for this is due to Dr. Richard 

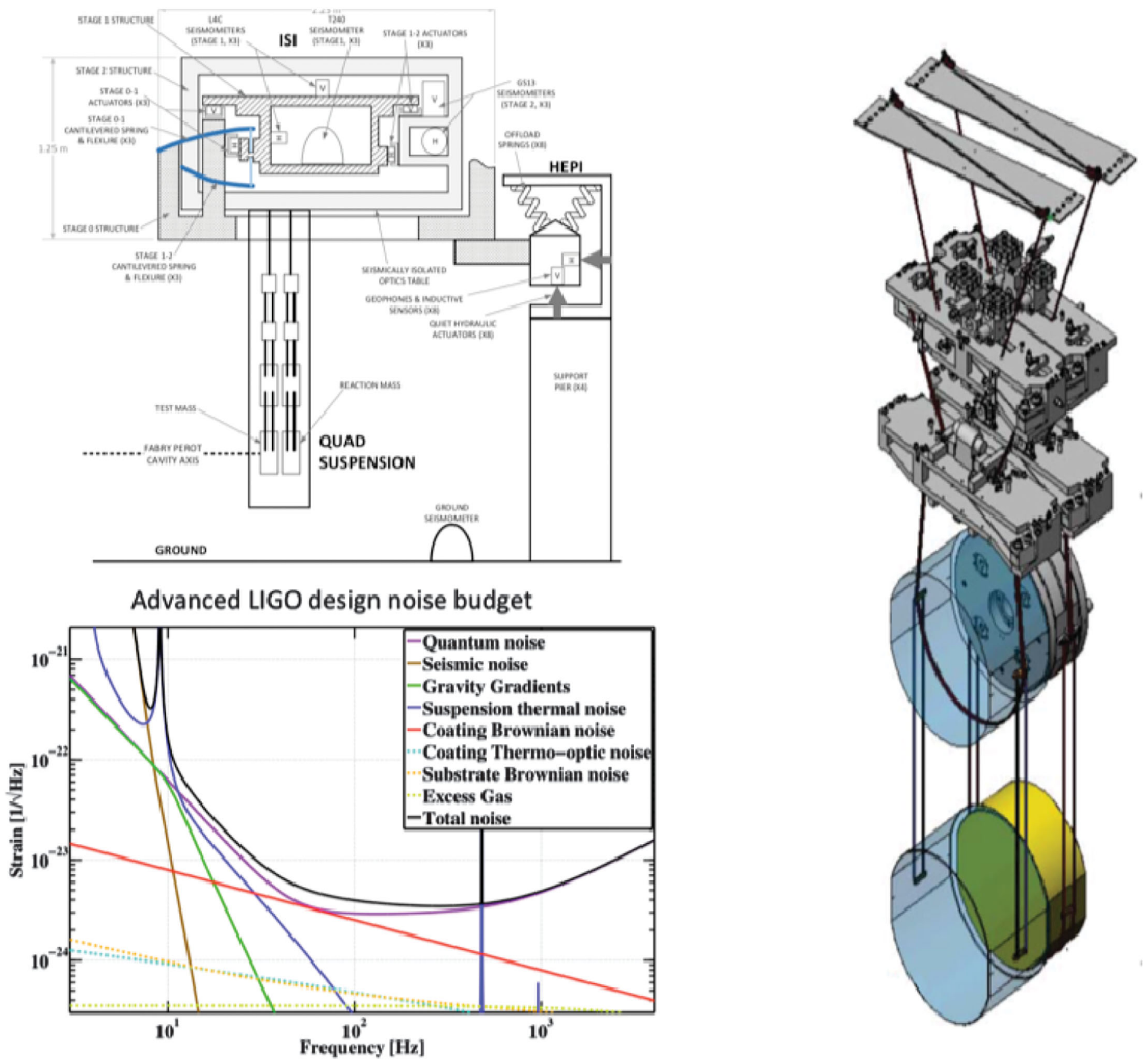

FIG. 16. Some properties of the advanced detector. Right: A quadruple pendulum system developed at Glasgow University which provides four stages of high frequency ground noise isolation as well as low pendulum thermal noise by using fused silica fiber supports (pioneered by Braginsky's Moscow group) in the final pendulum stage. Top left: A dual active seismic isolation system, initially developed at Stanford University, which becomes a platform for the quadruple suspension. The active system measures the motion of a platform with a three-dimensional seismometer and then feeds this back to a set of controllers to null the seismometer. Two such active systems are placed in series. Bottom left: The noise budget for the advanced detector. The dominant noise has become the quantum noise at both high and low frequencies (discussed in Kip's lecture). The noise limiting the performance at the sweet spot near $100 \mathrm{~Hz}$ has become the thermal noise generated in the mirror coatings. At this writing in February 2018, the noise in the advanced detector, as Barry will show, is about a factor 2.5 worse at the sweet spot than this projected noise curve.

Isaacson (Fig. 17), who was both an advocate for the field of gravitation within the Foundation and a strategist for the possible.

Once an internal NSF decision was made to construct LIGO, the strong support of three NSF Directors (Fig. 18) was essential to keep the project moving at critical junctures with Congress and amidst criticism in a skeptical subset of the scientific community.

A personal reflection: Science reporters who have written about the LIGO project have noted the 45 years from its inception to the first measurements. They comment on our perseverance and the novelty of a group of scientists working so long together without the satisfaction of a significant scientific result. This might be the way a person outside of the project views it. From the inside it is a very different story. What held the group together were the daily little triumphs or puzzles generated in the development of the ideas and technology (both hardware and software) as well as the collegiality of a dedicated group of people. 


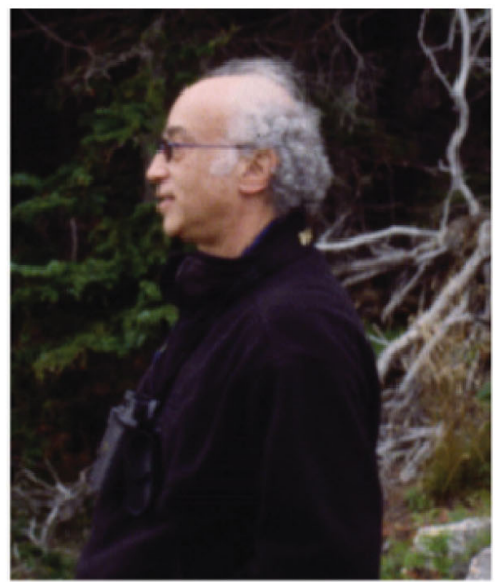
PHYSICAL REVIEW
VOLUME 166 , NUMBER
25 VEBRUARY 1968

Gravitational Radiation in the Limit of High Frequency.
II. Nonlinear Terms and the Effective Stress Tensor

Riamans A. Isuasson $\phi$

Department of Physics and Astronomy, Universily of Maryland, Colloge Parik, Maryland (Received 14 July 1967

The high-frequency expansion of a vacuam gravitational feekd in powers of its small wavelength is con. tinued. We go beyond the previously dliscussed linearization of the field equations to consider the lowestorder nonlinearities. These are shown to provide a matural, gauge-invariant, averaged stress tensor for the effective energe localized in the high-irequency gravitational waves. Under the asumption of the WKA netic null field. A Poynting vector is used to investigate the flow of energy and as that for an electromag waves, and it is seen that high-frequency waves propagate along null hypersurfaces and are not back. scattered by the lowest-order nonlinearities. Expressions for the total energy and momentum carried by the field to flat null infinity are given in terms of coordinate-independent hyperiarface integrals valid within regions of high field strength. The formalism is applied to the case of spherical gravitational waves where a

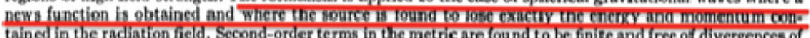

R. Isaacson (Gravitation at NSF)
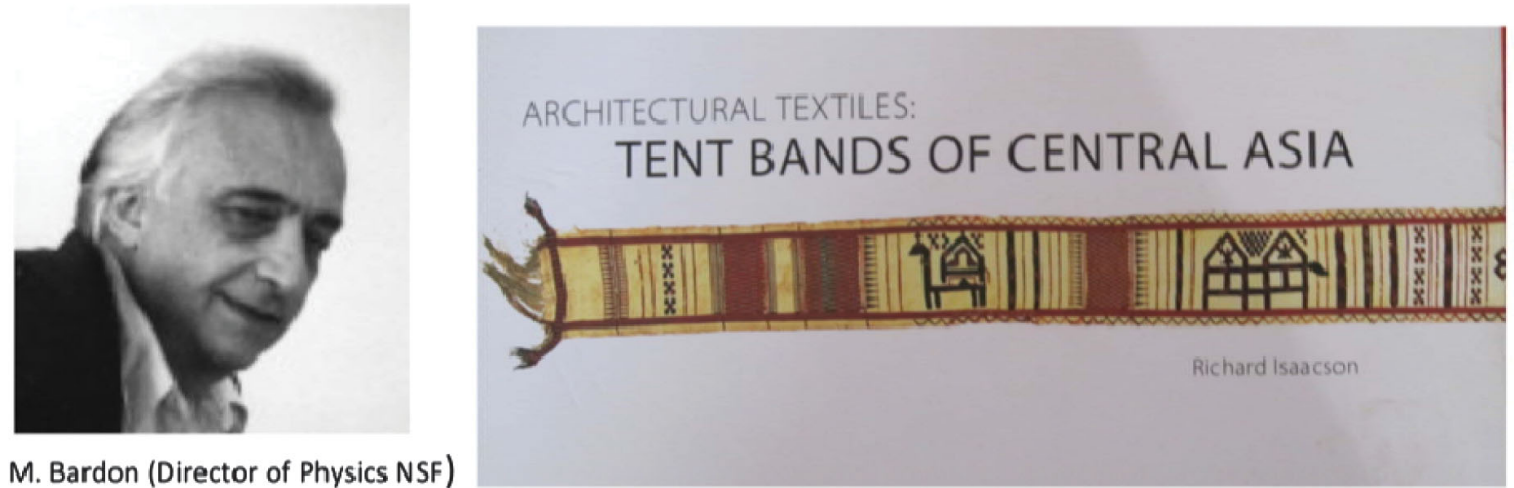

M. Bardon (Director of Physics NSF)

FIG. 17. A key individual at the National Science Foundation (NSF) was Dr. Richard Isaacson, who became discipline chief for Gravity in the Division of Physics in the mid-1970s. He had received his Ph.D. from the University of Maryland in Charles Misner's group and had written a paper that rigorously showed gravitational waves carried energy and momentum away from their sources-an issue that was still controversial in the 1960s. Isaacson sensed the scientific promise in a vital program of research in experimental and theoretical gravitation and became a powerful advocate for the field at the NSF. He convinced the Director of the Physics Division, Dr. Marcel Bardon, that gravitational wave detection, although risky since the technology needed development and the sources were not certain, was the type of science which could produce transformative results and was well suited to the NSF. In addition to his advocacy within the Foundation, Isaacson guided the scientists in the field with strategic advice and wisdom about what was possible. After he retired from the NSF, he made a profession of a significant hobby in a study of textiles and the cultures that produce them in Asia. He curated an exhibit of the tent bands of yurts for the Washington Textile Museum.

\section{NSF Directors critical for LIGO}

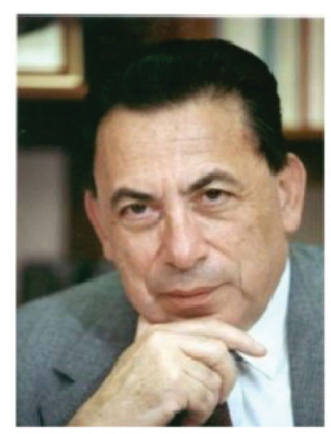

Eric Block

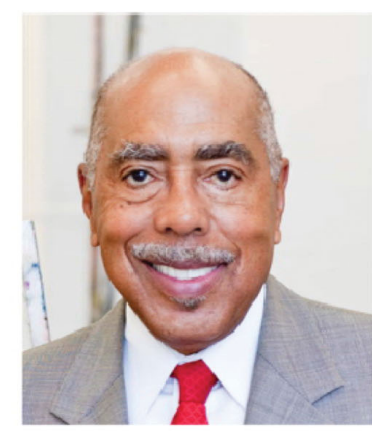

Walter Massey

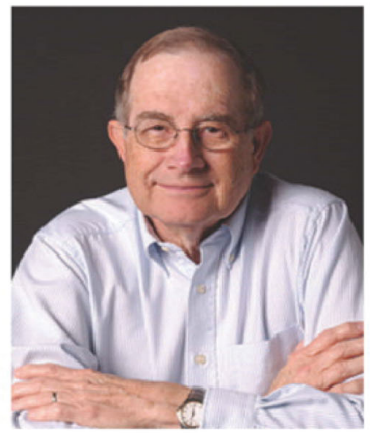

Neal Lane

FIG. 18. Three Directors of the National Science Foundation who played critical roles in the evolution of the LIGO project: Eric Block was the first Director to consider going forward with the large baseline LIGO project. Walter Massey defended the program during the time when astronomers considered LIGO premature and a possible waste of funds. Neal Lane was a strong advocate for a line item in the congressional NSF budget to support Major Research Equipment Funds for Construction (MREFC). LIGO was one of the first projects to take advantage of these funds, and since then the funds have been used to begin many other large NSF projects. 


\section{REFERENCES}

Brault, J., 1963, “Gravitational Redshift of Solar Lines,” Bull. Am. Astron. Soc. 8, 28.

Drever, R. W. P., 1983, "Interferometric detectors for gravitational radiation," Gravitational Radiation, NATO Advanced Physics Institute, Les Houches, edited by N. Deruelle and T. Piran (North-Holland Publishing, Amsterdam), p. 321.

Einstein, A., 1915, "Zur Allgemeinen Relativitätstheorie," Sitzunsberichte der Königlich Preussichen Akademie der Wissenschaften, pp. 778, 799 [https://einsteinpapers.press.princeton.edu].

Einstein, A., 1916a, "Die Grundlage der Allgemeinen Relativitätstheorie," Ann. Phys. (Berlin) 354, 769.

Einstein, A., 1916b, "Näherungsweise integration der feldgleichungen der gravitation," Sitzungsber. Preuss. Akad. Wiss. Berlin (Math.Phys.), pp. 688-696.

Einstein, A., 1918, "Über gravitationswellen," Sitzungsber. Preuss. Akad. Wiss. Berlin (Math. Phys.), pp. 154-167.

Fomalont, E. B., and R. A. Sramek, 1975, "A Confirmation of Einstein's General Theory of Relativity by Measuring the Bending of Microwave Radiation in the Gravitational Field of the Sun," Astrophys. J. 199, 749.

Gertsenshtein, M. E., and V. I. Pustovoit, 1963, "On the Detection of Low Frequency Gravitational Waves," Sov. Phys. JETP 16, 433.

Linsay, Paul, Peter Saulson, Rainer Weiss, and Stan Whitcomb, 1983, NSF Bluebook, https://emvogil-3.mit.edu/ weiss/ligo_history_ documents/NSF_bluebook_1983.pdf.

Meers, B. J., 1988, "Recycling in laser-interferometric gravitationalwave detectors," Phys. Rev. D 38, 2317.
Pirani, F. A.E., 1956, "On the physical significance of the Riemann tensor," Acta Phys. Pol. 15, 389; 1957, "Measurement of classical gravitation fields," Chapel Hill Conference (Reference 9, 61).

Pound, R. V., and G. A. Rebka, 1960, "Apparent Weight of Photons," Phys. Rev. Lett. 4, 337.

Rickles, Dean, and Cecil M. DeWitt, 1957, Eds., "The Role of Gravitation in Physics," Report from the 1957 Chapel Hill Conference, Reprinted by Max Planck Research Library for the History and Development of Knowledge Sources 5, submitted by Jurgen Renn, Alexander Blum, and Peter Damerow.

Shoemaker, D., R. Schilling, L. Schnupp, W. Winkler, K. Maischberger, and A. Rüdiger, 1988, "Noise behavior of the Garching 30-meter prototype gravitational wave detector," Phys. Rev. D 38, 423.

Taylor, J.H., and J.M. Weisberg, 1982, "A new test of general relativity: Gravitational radiation and the binary pulsar PSR 1913 +16," Astrophys. J. 253, 908.

Vogt, Rochus E., Ronald W. P. Drever, Frederick J. Raab, Kip S. Thorne, and Rainer Weiss, 1989, Caltech/MIT NSF proposal, https://dcc.ligo.org/LIGO-M890001/public/main/.

Weber, J., 1960, "Detection and generation of gravitational waves," Phys. Rev. 117, 306.

Weber, J., 1969, "Evidence for the Discovery of Gravitational Waves," Phys. Rev. Lett. 22, 1320.

Weiss, R., 1972, "Electromagnetically coupled broadband gravitational antenna," Research Laboratory for Electronics MIT, Quarterly Progress Report, No. 105, 54. 